\title{
Fuzzy Control and Virtual Instrumentation for Superheated Steam Electrolysis in Solid Oxide Cells
}

\author{
Álvaro Aguinaga ${ }^{1}$, Mauricio Duque ${ }^{1}$, Fredy Llulluna ${ }^{1}$, Telmo Moreno ${ }^{2}$, Nelson Jara ${ }^{3}$ \\ ${ }^{1}$ Department of Mechanical Engineering, Escuela Politécnica Nacional \\ Ladrón de Guevara E11-253, Quito, Ecuador \\ alvaro.aguinaga@epn.edu.ec; mauricio.duque@epn.edu.ec; fredy.llulluna@epn.edu.ec \\ ${ }^{2}$ Department of Mechanical Engineering, Escuela Superior Politécnica del Chimborazo \\ Panamericana Sur km 11/2, Riobamba, Ecuador \\ telmomoreno@hotmail.com \\ ${ }^{3}$ Department of Mechanical Engineering, Universidad Politécnica Salesiana \\ Calle Vieja 12-30 y Elia Liut, Cuenca, Ecuador \\ njara@ups.edu.ec
}

\begin{abstract}
At the Escuela Politécnica Nacional, University of Technology from Quito-Ecuador, it is researching and developing a prototype of a Solid Oxide Electrolyser Cell (SOEC), for the production of hydrogen by electrolysis of superheated steam. Traditional methods of automatic control and instrumentation have not proved effective in this case, the high complexity of the processes and the nonlinear characteristics of the variables involved, therefore it is necessary to use new forms of intelligent control, such as fuzzy control and equally high-tech instrumentation as virtual instrumentation. With this goal has been developed and installed in the SOEC a fuzzy controller, which requires action of a fuzzifier on the input variables, then is defined a set of fuzzy logic rules which is known as the fuzzy inference system or reasoning; and finally the action of a defuzzifier on the output variables resulting. The fuzzy inference system must produce the greatest amount of hydrogen in the shortest time. It has also been developed and installed in the SOEC a virtual instrumentation system based on open source platform, developed by the authors of this research. The respective probes were installed for the input variables and output variables. Has been performed the Programming of the open source platform; and, was installed and programmed a HMI (Human Machine Interface) to process controlling and monitoring with a graphics-based visualization, thereby achieving provide virtual instrumentation for the SOEC. It has demonstrated the high effectiveness of implementing a fuzzy controller and virtual instrumentation for the control and real-time monitoring of the processes occurring in the SOEC. These installed systems will be essential to the phases of: parametric sensitivity analysis and validation of the prototype of SOEC system, which is being researched and developed. The state of the art and perspectives of these methods are also evaluated.
\end{abstract}

Keywords: hydrogen, SOEC, electrolysis, superheated steam, fuzzy, VI, HMI.

\section{Introduction}

The dramatic decline in world oil reserves will in a few years, if no solution is found, an unprecedented energy crisis that would force drastically change the present mode of life. The current energy situation, with problems of pollution, climate change, international conflicts motivated by access to resources, and depletion of fossil fuels, requires new technologies. These include the hydrogen, which many assigned the role of displacing fossil fuels to get to base our future in "hydrogen economy". [1], [2]

For the next years, hydrogen as a fuel is considered as a potential source of renewable energy, which avoid further deterioration of our environment, that it is used in processes of combustion or to regenerate electricity in fuel cells. It appears that the future of energy passing through hydrogen, the cleanest fuel there is. It is versatile and very effective. A revolutionary fuel because it will transform the social and economic relationships around the world. It also means hope in the conquest of a sustainable energy economy. [3]

Hydrogen production via high temperature steam electrolysis is a promising technology as it involves less electrical energy consumption compared to conventional low temperature water electrolysis, as consequence of the more favorable thermodynamic and electrochemical kinetic conditions for the reaction. [4] The total energy required $(\Delta \mathrm{H})$ for dissociation, either water or steam, is the sum of the Gibbs free energy $(\Delta \mathrm{G})$ and a certain amount of heat energy. The demand for 
electricity $(\Delta \mathrm{G})$, decreases with increasing temperature. For this reason, steam electrolysis can produce hydrogen with less electrical power than is required for the case of water at lower temperatures. [5]

Precisely in the Department of Mechanical Engineering of the Escuela Politécnica Nacional, University of Technology from Quito-Ecuador, is developing the Solid Oxide Electrolyser Cell (SOEC) for hydrogen production by high temperature steam electrolysis. In the Fig. 1, the components and operation of the Solid Oxide Electrolyser Cell (SOEC) that is being developed, is presented schematically [4], [6]; and in Fig. 2 a photograph the construction progress of the prototype is presented.

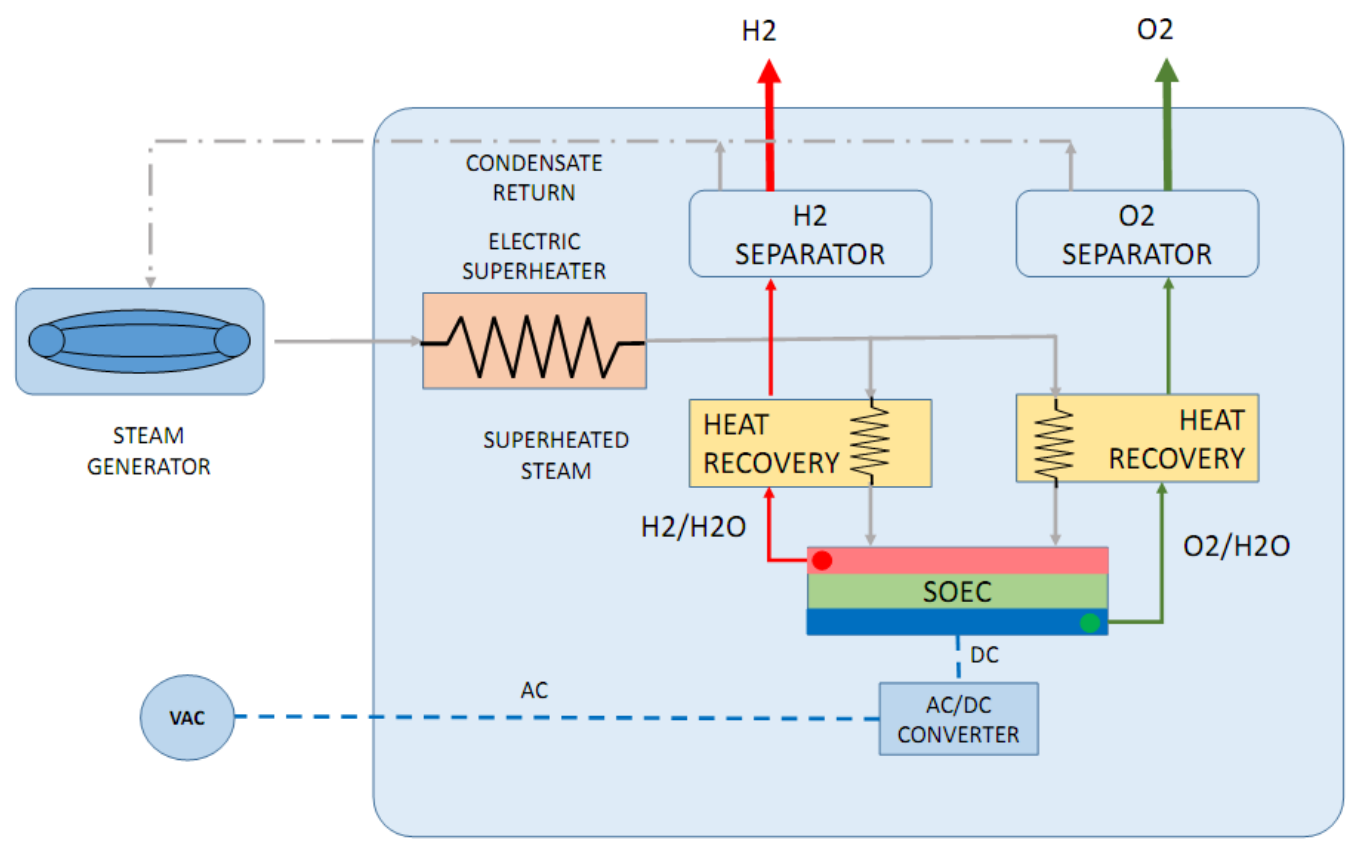

Fig. 1: Scheme of a Solid Oxide Electrolyser Cell (SOEC) that is being developed.

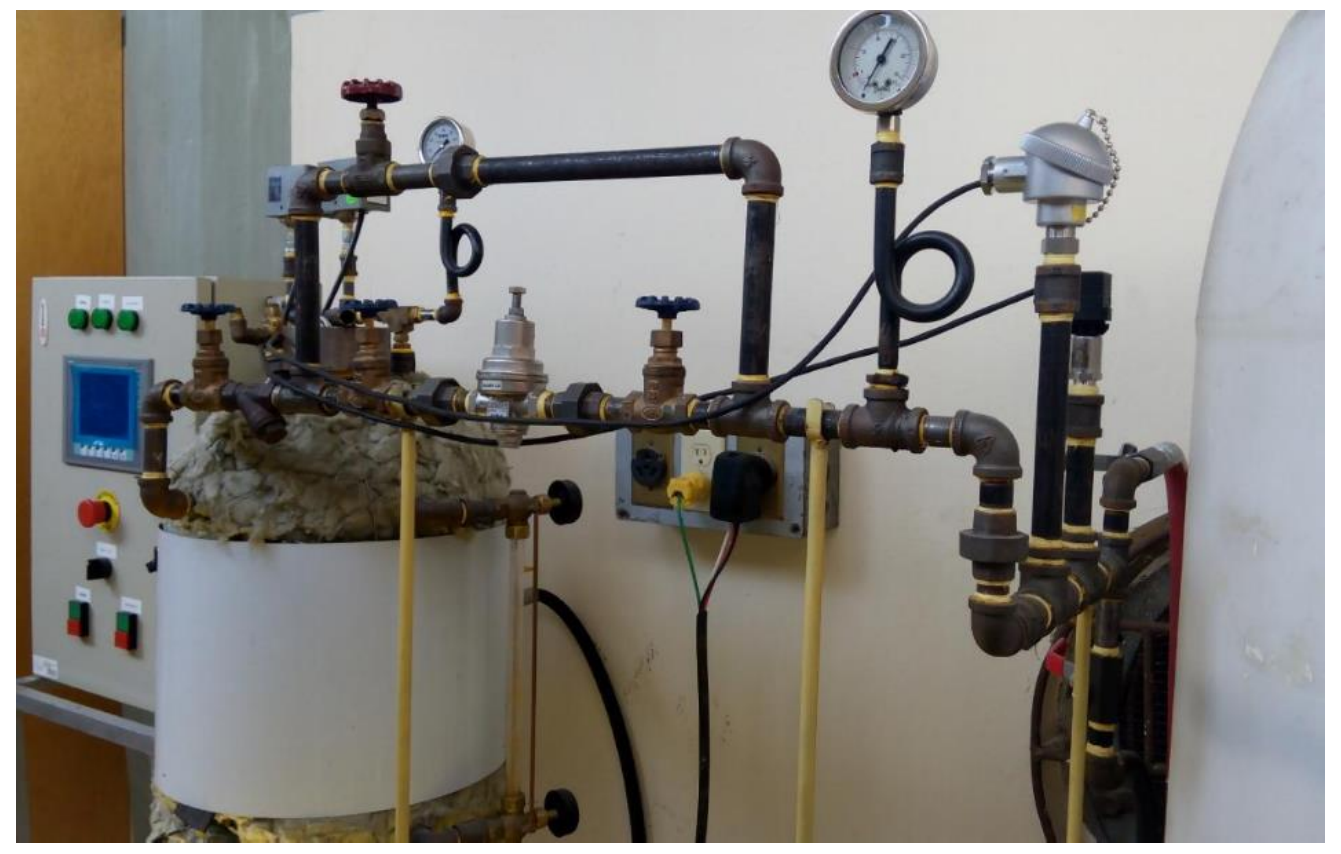

Fig. 2: Photograph the construction progress of the prototype SOEC. 
It was determined the Solid Oxide Electrolyser Cell performance as function a lot of the operating parameters: temperature, pressure, current density, electrolyte resistance and others. In the development of the SOEC, there have been some problems that have motivated the development of several research projects associated, such is the case of the control and instrumentation of the SOEC. Traditional methods of automatic control and instrumentation have not proved effective in this case, the high complexity of the processes and the nonlinear characteristics of the variables involved, therefore it is necessary to use new forms of intelligent control, such as fuzzy control and equally high-tech instrumentation as virtual instrumentation. Precisely this problem of research and the results obtained on the development of a fuzzy controller with virtual instrumentation for the SOEC is what is reported in this paper.

\section{Fuzzy control for the SOEC}

The development of fuzzy logic based controller, provides solutions and shorten the period of development control processes, what due to their complexity involving a highly nonlinear mathematical model, which is why their control with standard tools would be highly inaccurate and unstable and feedback because of excessively high response times of the controller. [7]

A fuzzy controller, is a knowledge-based structure defined by a set of rules or fuzzy inferences, which contain a fuzzy quantification expert linguistic description of how to perform a proper control. The fuzzy system is necessarily part of a technical system that works with numeric values (signals), with no fuzzy values, inputs and outputs and in general are considered two types of fuzzy systems:

a) Takagi-Sugeno fuzzy controller that their inputs and outputs are variables with real values. [8]

b) Mandami fuzzy controller interposes a fuzzifier for the input variables and a defuzzifier for the output variables. [8] This principle is illustrated in the Fig. 3.

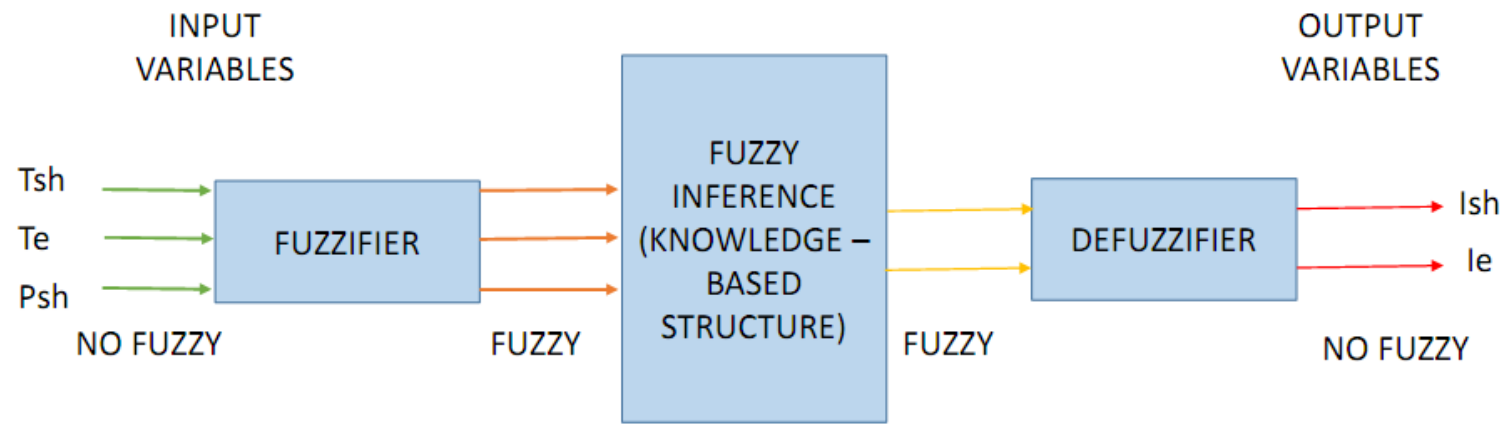

Fig. 3: Scheme of a Mandami fuzzy controller.

Fuzzifier of the input variables determines the degree of satisfaction for the linguistic values (membership functions of fuzzy set) of the linguistic variables are set to the input values not fuzzy. Such fuzzifier determined that "a variable degree is good". This is done using the membership function. This degree is called "satisfaction or compliance with part "IF". [9]

For each rule in the fuzzy inference, the degree of satisfaction of the "THEN" part is formed from the satisfaction of the IF part by a certain method. This process is also called Implication. The degree of satisfaction of the part "THEN" is equivalent to the satisfaction of the rule, which is also called intensity rule. All these assessments of individual rules are put together in a membership function for the output signal, which is also called composition. [10]

The resulting output variables are membership functions of fuzzy set, that there must be defuzzifier. The most used defuzzifier techniques are: [9]
a) WAM Weighted Averange Method.
b) COA o Center of Areas.
c) COS o Center of Sums.
d) MOM o Mean of Maximum.
e) CA o Center Average. 


\subsection{Fuzzy controller design for the SOEC}

The design of the fuzzy controller is based on the model of Mamdani fuzzy controller, which requires action of a fuzzifier on the input variables, then is defined a set of fuzzy logic rules which is known as the fuzzy inference system or reasoning; and finally the action of a defuzzifier on the output variables resulting. The fuzzy inference system must produce the greatest amount of hydrogen in the shortest time. The components of the fuzzy control process for the production of hydrogen from SOEC, shown in Fig. 4:

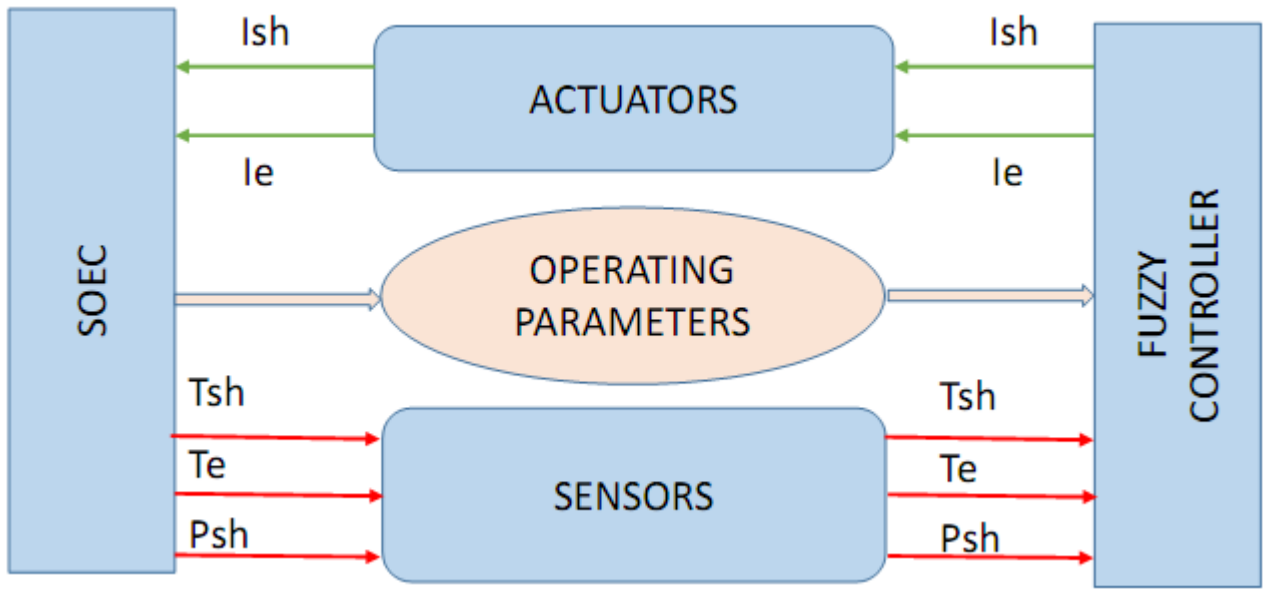

Fig. 4: Components of the fuzzy control process for the production of hydrogen from SOEC.

In the scheme of Fig. 4 are presented the input and output variables of the fuzzy controller, there are synthesized from a detailed analysis. These variables are:

a) Input variables: temperature of superheated steam (Tsh), pressure superheated steam (Psh) and SOEC temperature $(\mathrm{Te})$.

b) Output variables: current of the electric heater (Ish) and SOEC current (Ie).

Of course, the process has involved several other operational variables that will be characterized in the section of virtual instrumentation, but at this point only interest those involved in the action of control.

\subsection{Fuzzifier, rules in the fuzzy inference and defuzzifier}

The first step consists of fuzzification of the three input variables (Tsh, Psh and Te), defining its domain, establishing upper and lower limits, for this case the range of the input variables are set percentage between $0 \%$ and $100 \%$. The next step is to define the number and type of membership functions. The membership functions used here are two trapezoidal and one triangular, because its structure allows better define the linguistic value associated with the temperature and pressure are also simple, to better driving ranges and generating less computational expense.

The second step consists of fuzzification of the two output variables (Ish and Ie), for this case the range of the input variables are set percentage between $0 \%$ and $100 \%$ too. The membership functions used here are five triangular.

In this case are defined the rules in the fuzzy inference as the particular correspondence between two-valued and multivalued logical operations for AND, OR, and NOT. This correspondence is by no means unique.

Fuzzy sets and fuzzy operators are the subjects and verbs of fuzzy logic. These if-then rule statements are used to formulate the conditional statements that comprise fuzzy logic. A single fuzzy if-then rule assumes the form if $\mathrm{x}$ is $\mathrm{A}$ then $\mathrm{y}$ is $\mathrm{B}$ where $\mathrm{A}$ and $\mathrm{B}$, are linguistic values defined by fuzzy sets on the ranges (universes of discourse) $\mathrm{X}$ and $\mathrm{Y}$, respectively. The if-part of the rule " $\mathrm{x}$ is $\mathrm{A}$ " is called the antecedent or premise, while the then-part of the rule " $\mathrm{y}$ is B" is called the consequent or conclusion. An example of such rules might be:

IF Tsh is A and Psh is B or Te is C THEN Ish is D and Ie is E.

In the diagram in Fig. 5, the fuzzy control system developed is presented. 


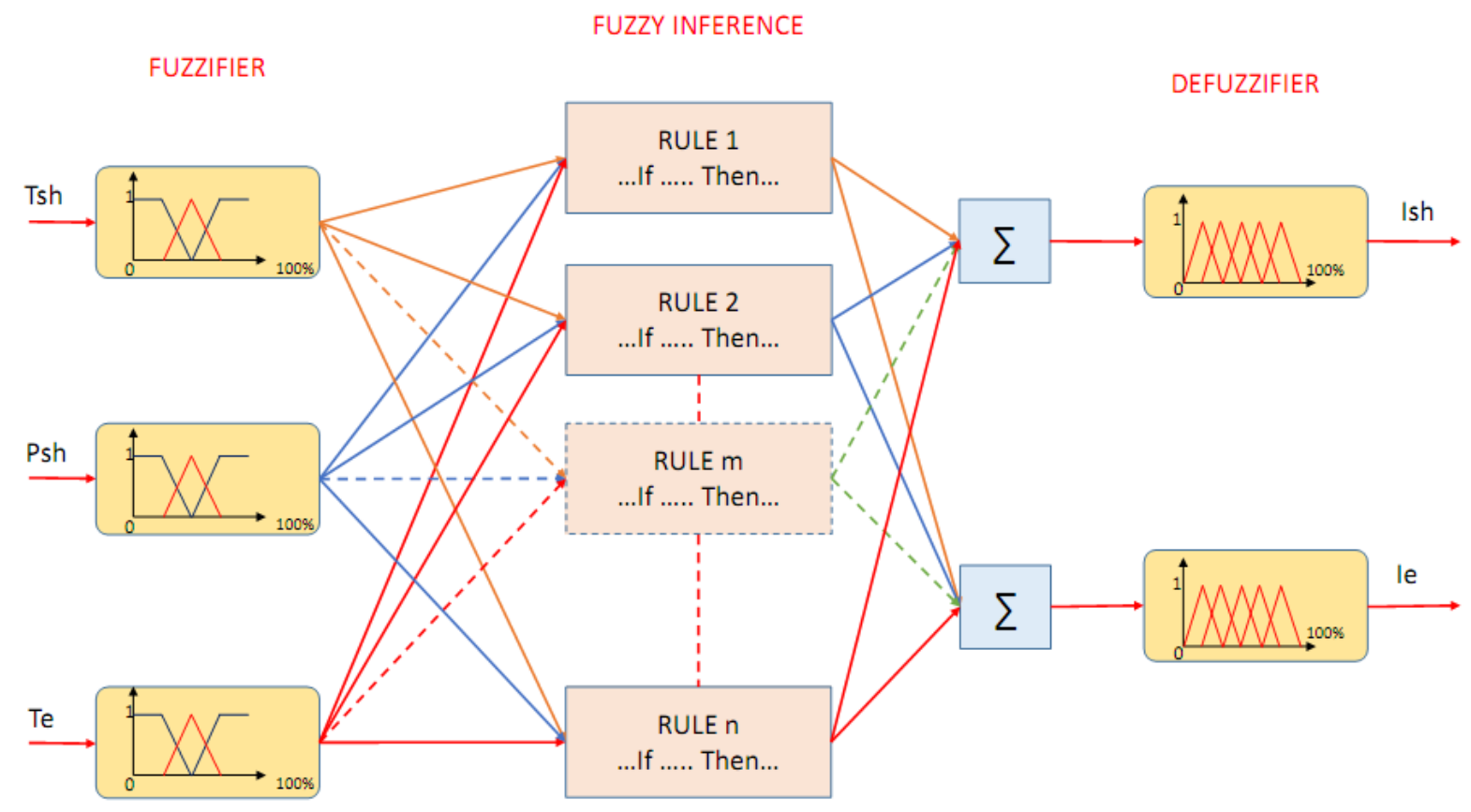

Fig. 5: The fuzzy control system developed.

For the analysis, synthesis and optimization of fuzzy controller model, it used the fuzzy Logic Toolbox, Matlab [11], getting an optimized model was validated with multiple simulations in the indicated toolbox.

Based on the required operating conditions, proceeding to program the optimized model of fuzzy controller in the PLC, developing the platform necessary for the creation of the fuzzy controller (fuzzification, creation and evaluation rules and defuzzification) functions. Since the program conditions require perform activations comparisons, escalations and calculation equations, we chose to use the ladder language.

\section{Virtual instrumentation (VI)}

Virtual instrumentation is a concept introduced by the company National Instruments (2001), developing the system LabVIEW from National Instruments. The most simple and most favorite definition of virtual instrumentation (VI) is that VI replaces traditional or specific physical measurement devices by software module with universal I/O interface. It is also common to use interfaces HMI (Human Machine Interface). The user interface in a manufacturing or process control system provides a graphics-based visualization of an industrial control and monitoring system. [12]

A virtual instrument can perform three basic functions of a conventional instrument: acquisition, analysis and presentation of data. However, the virtual instrument allows to customize the instrument and add much more functionality without incurring additional costs, therefore an instrument may provide one or more measurements of the same physical phenomenon. Additionally, the concept of "virtual instrumentation" goes beyond the simple measurement of current or voltage. It also involves the processing, analysis, storage, distribution and display of data and information related to the measurement of one or more specific signals. With these, using software that allow the implementation of control algorithms, it is possible to integrate and control complex processes. That is, the virtual instrument does not conform to the acquisition of the signal, but also involves the man-machine interface, the functions of analysis and signal processing. [13], [14]

Similarly, currently it is developing the Research Project CEPRA 2015, of CEDIA (National Research and Education Network of Ecuador), with the collaboration of Department of Mechanical Engineering of the Escuela Politécnica Nacional, University of Technology of Ecuador and others authors of this research. In this research project, it is developing and implementing a platform open source for a remote laboratory virtual instrumentation, accessible via the Internet, open source, focused on low budget. 
To implement the virtual instrumentation to the Solid Oxide Electrolyser Cell, was used the same open source platform, developed from the research project referred. The respective probes were installed for the three input variables (Tsh, Psh and Te) and the two output variables (Ish and Ie), at sites corresponding in the SOEC. Has been performed the Programming of the open source platform; and, was installed and programmed a HMI (Human Machine Interface) to process controlling and monitoring with a graphics-based visualization, thereby achieving provide virtual instrumentation for the SOEC.

\section{Installing the fuzzy controller and virtual instrumentation for the SOEC}

This platform open source and the virtual instrumentation, with the respective sensors, actuators, HMI and fuzzy controller, are installed on the system Solid Oxide Electrolyser Cell (SOEC). In the Fig. 6, it can be seen the fuzzy controller and VI implemented.

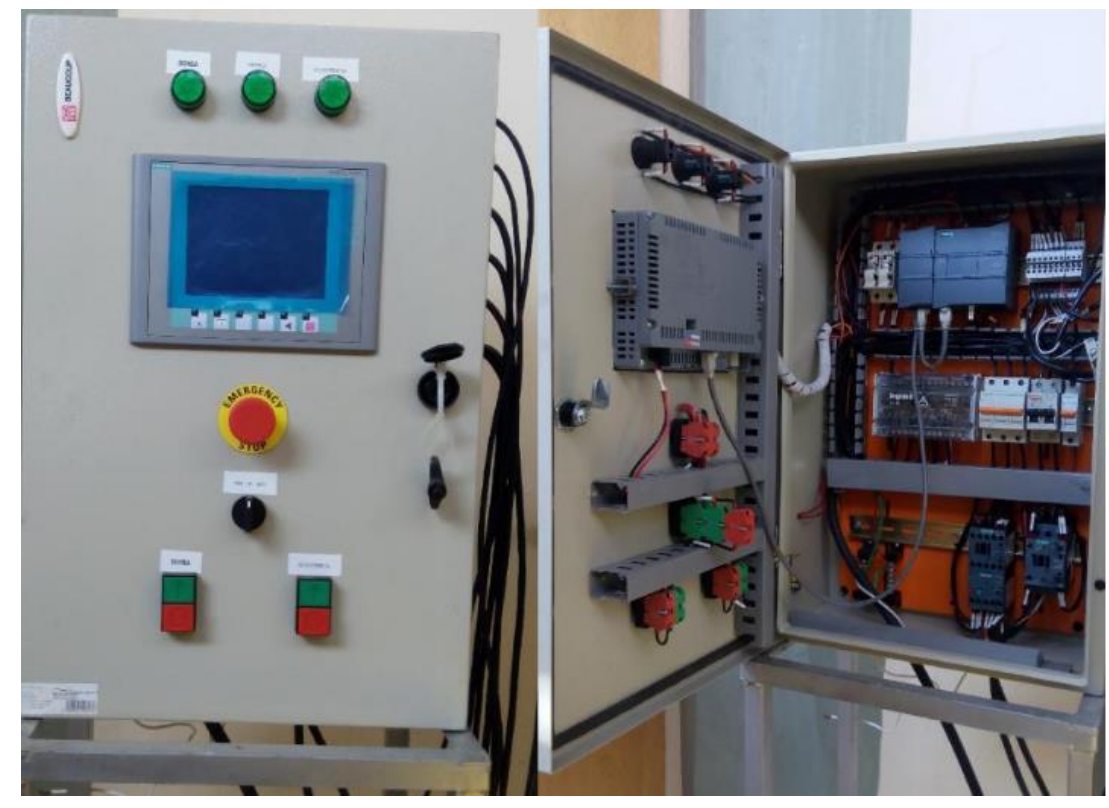

Fig. 6: Fuzzy controller and VI implemented.

With these installed systems are performing the first test operation of the system, which has proved the high effectiveness of the fuzzy controller and virtual instrumentation for the control and real-time monitoring of the processes occurring in the Solid Oxide Electrolyser Cell.

Thanks to these systems installed, is being performed trials and testing to conclude the validation stage of the Solid Oxide Electrolyser Cell (SOEC) model, perform a sensitivity analysis of parameters and optimize the prototype built.

\section{Conclusions}

- Traditional methods of automatic control and instrumentation are not effective for supervision and control of systems a Solid Oxide Electrolyser Cell (SOEC), mainly because of the high complexity of the processes and the nonlinear characteristics of the variables involved equations model.

- It has been developed and installed in the SOEC a fuzzy controller, that has a fuzzifier on the input variables, is defined a set of fuzzy logic rules which is known as the fuzzy inference system, and finally the action of a defuzzifier on the output variables resulting.

- The respective probes were installed for the input variables and output variables. Has been performed the Programming of the open source platform; and, was installed and programmed a HMI to process controlling and monitoring with a graphics-based visualization, thereby achieving provide virtual instrumentation for the SOEC. 
- It has demonstrated the high effectiveness of implementing a fuzzy controller and virtual instrumentation for the control and real-time monitoring of the processes occurring in the Solid Oxide Electrolyser Cell.

- These installed systems will be essential to the phases of: parametric sensitivity analysis and validation of the prototype of SOEC system, which is being researched and developed.

- It is necessary to continue studying the state of the art and evaluating the prospects of these methods by the importance to replace fossil fuels with sustainable use of new and innovative technologies.

\section{Acknowledgements}

Our special thanks go to CEDIA (National Research and Education Network of Ecuador), Research Project CEPRA

2015, for the financial and cooperation for the development of this Research Project.

\section{References}

[1] A. Aguinaga, "Design and construction of a prototype plant of domestic hydrogen dispensing with hybrid electrolyzer, solar and electric," Escuela Politécnica Nacional, Polytechnic Journal, vol. 30, no.1, 2009, ISSN: 13900129.

[2] T. Tabazah, "Utilization of water produced hydrogen for domestic uses," Int. Journal of Thermal \& Environmental Engineering, vol. 7, no. 2, pp. 95-99, 2014.

[3] T. Litardo, F. Lllulluna, A. Aguinaga, Odebrecht, "Development of sustainable cells H2Exergia for using hydrogen as vector of energy generated from solar energy," Award for Sustainable Development of Ecuador 2013, ISBN 9789942-13-745-6, 2013.

[4] A. Brisse, J. Schefold, M. Zahid, "High temperature water electrolysis in solid oxide cells," International Journal of Hydrogen Energy, vol. 33, no. 20, pp. 5375-5382, October 2008, ISSN 0360-3199.

[5] G. Schiller, A. Ansar, M. Lang, "High temperature water electrolysis using metal supported solid oxide electrolyser cells (SOEC)," Journal of Applied Electrochemistry, vol. 39, no. 2, pp. 293, 2009.

[6] M.A. Laguna-Bercero, "Recent advances in high temperature electrolysis using solid oxide fuel cells: A review," Journal of Power Sources, vol. 203, pp. 4-16, 1 April 2012, ISSN 0378-7753.

[7] D. Drianko, H. Hellendoorn, and M. Reinfrank, "An introduction to fuzzy control," Springer Science \& Business Media, 2013.

[8] H. T. Nguyen and M. Sugeno, (Eds.). Fuzzy systems: modeling and control, vol. 2, 2012.

[9] R. R. Yager and L. A. Zadeh, (Eds.). "An introduction to fuzzy logic applications in intelligent systems," Springer Science \& Business Media, vol. 165, 2012.

[10] I. S. Shaw, Fuzzy control of industrial systems: theory and applications, Springer, vol. 457, 2013.

[11] Matlab, "Fuzzy Logic Toolbox", 2010, The MathWorks, Inc.

[12] M. Branzila, C. Alexandru, C. Donciu, A. Trandabăț, and C. Schreiner, "Virtual environmental measurement center based on remote instrumentation," Environmental Engineering and Management Journal, vol.6, no.6, pp. 517-520, 2007.

[13] M.S. Farias, P.V.R. Carvalho, F. Lacerda, P. Pedrosa, and C. S. Borges, "Development of virtual instrumentation on mobile devices," Instituto de Engenharia Nuclear: Progress Report, (1), 44, 2013.

[14] L. B. G. Campanhol, S. A. O. Silva, and A. Goedtel, "Virtual instrumentation applied to calculation of electrical power quantities in single-phase systems," Proc. ICREPQ'12, 2012. 